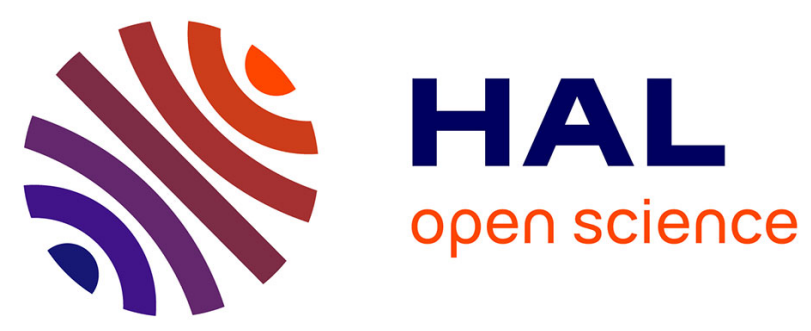

\title{
Exploiting the Phase in Diffusion MRI for Microstructure Recovery: Towards Axonal Tortuosity via Asymmetric Diffusion Processes
}

Marco Pizzolato, Demian Wassermann, Timothé Boutelier, Rachid Deriche

\section{- To cite this version:}

Marco Pizzolato, Demian Wassermann, Timothé Boutelier, Rachid Deriche. Exploiting the Phase in Diffusion MRI for Microstructure Recovery: Towards Axonal Tortuosity via Asymmetric Diffusion Processes. Medical Image Computing and Computer Assisted Intervention, 2015, Munich, Germany. 10.1007/978-3-319-24553-9_14 . hal-01152672

HAL Id: hal-01152672

https://hal.inria.fr/hal-01152672

Submitted on 19 May 2015

HAL is a multi-disciplinary open access archive for the deposit and dissemination of scientific research documents, whether they are published or not. The documents may come from teaching and research institutions in France or abroad, or from public or private research centers.
L'archive ouverte pluridisciplinaire HAL, est destinée au dépôt et à la diffusion de documents scientifiques de niveau recherche, publiés ou non, émanant des établissements d'enseignement et de recherche français ou étrangers, des laboratoires publics ou privés. 


\title{
Exploiting the Phase in Diffusion MRI for Microstructure Recovery: Towards Axonal Tortuosity via Asymmetric Diffusion Processes
}

\author{
Marco Pizzolato ${ }^{1, \star, \star \star}$, Demian Wassermann ${ }^{1, \star}$, \\ Timothé Boutelier ${ }^{2}$, and Rachid Deriche ${ }^{1}$ \\ 1 Athena, Inria Sophia Antipolis - Méditerranée, France \\ \{marco.pizzolato, demian.wassermann, rachid.deriche\}@inria.fr \\ 2 Olea Medical, La Ciotat, France \\ timothe.boutelier@olea-medical.com
}

\begin{abstract}
Microstructure recovery procedures via Diffusion-Weighted Magnetic Resonance Imaging (DW-MRI) usually discard the signal's phase, assuming symmetry in the underlying diffusion process. We propose to recover the Ensemble Average Propagator (EAP) directly from the complex DW signal in order to describe also eventual diffusional asymmetry, thus obtaining an asymmetric EAP. The asymmetry of the EAP is then related to tortuosity of undulated white matter axons, which are found in pathological scenarios associated with axonal elongation or compression. We derive a model of the EAP for this geometry and quantify its asymmetry. Results show that the EAP obtained when accounting for the DW signal's phase provides useful microstructural information in such pathological scenarios. Furthermore, we validate these results insilico through 3D Monte-Carlo simulations of white matter tissue that has experienced different degrees of elongation/compression.
\end{abstract}

Keywords: diffusion MRI, phase, EAP, asymmetry, tortuosity, axon

\section{Introduction}

A rather unexplored field in Diffusion-Weighted Magnetic Resonance Imaging (DW-MRI) is the use of the phase component of the signal to quantify white matter microstructural characteristics. Despite the DW signal's complex nature, current approaches discard the phase and characterize microstructure using just the signal's magnitude [1-3]. In this paper, we show that the signal's phase component plays an important role in microstructure characterization. It provides a novel approach towards the quantification of diffusion properties. Particularly, we exploit the signal's phase to quantify different levels of tortuosity in undulated axons within a voxel, which can be associated with pathology through, for instance, axonal compression.

^ Marco Pizzolato and Demian Wassermann contributed equally to this work.

** The author expresses his thanks to Olea Medical and the Provence-Alpes-Côte d'Azur (PACA) Regional Council for providing grant and support for this work. 

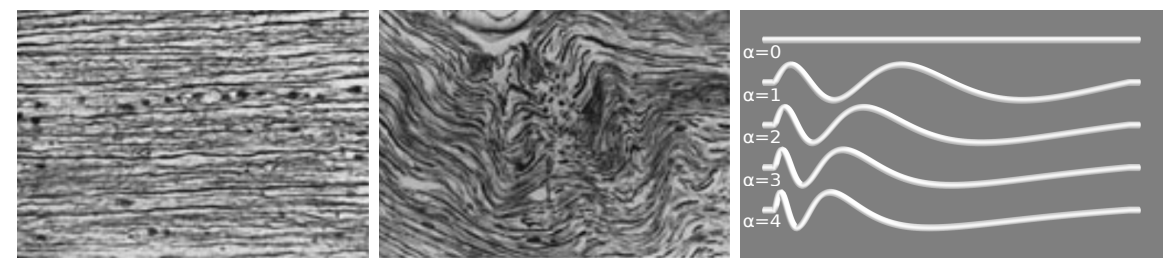

Fig. 1. From left to right: elongated and compressed WM tissue, reproduced with permission from [5]; straight/undulated axonal models for different tortuosity rates $\alpha$.

White matter (WM) tissue may present different microstructural organizations depending on its nature and pathological condition. For instance, axons composing the tissue can be straight as shown in fig. 1 left. However axons may appear sinusoidal, and pathological conditions such as those caused by cervical cord injury can result in changes of undulation amplitude and tortuosity due to longitudinal elongation or compression $[4,5]$, as shown in central fig. 1 . The movement of spins within the WM tissue is influenced by the type of organization. Thus, a profound knowledge of the diffusion process can give insights into its microstructural properties.

DW-MRI allows to probe diffusion in a tissue sample by measuring a complexvalued signal related to the displacement probability of spins. The average displacement probability of the ensemble of spins contained within a voxel is known as Ensemble Average Propagator (EAP), and is related to the signal attenuation via an inverse Fourier transform [6]. Tissue microstructure influences spins displacements. Hence, EAP and DW signal can be exploited to measure microstructural properties. Recent work [4] proves that undulated axons, modeled as sinusoids, are a confound on axonal diameter estimation from DW images.

We show that by exploiting the DW signal's phase it is possible to characterize axonal tortuosity, which can be linked to elongation/compression phenomena in pathological scenarios. Compressed axons are partially convoluted and show irregular undulation with a non-uniform tortuosity along the longitudinal direction (see fig. 1 right). Hence, we model the compressed axon as a sinusoid with varying tortuosity, inducing asymmetry in its shape. Moreover, as the degree of compression increases, the tortuosity variation rate along the axon also increases. Spins diffusing within these axons undergo an asymmetric diffusion process resulting in an informative DW signal's phase, sensible to increasing tortuosity rates. The amount of diffusion asymmetry thus gives important insights into the underlying degree of compression.

Diffusion process asymmetries have already been investigated [7]. However, current approaches to obtain the EAP only use the DW magnitude signal and assume axial symmetry along each diffusion direction. We show that, by exploiting the DW signal's phase component, we recover an EAP describing also asymmetric diffusion which asymmetry is linked to the axonal tortuosity rate, and thus compression. To prove this, we first derive an analytic model of the EAP corresponding to the proposed axonal geometry, and obtain the correspondent DW 
complex signal. Using the complex signal we recompute the EAPs for increasing tortuosity rates, corresponding to scenarios of increasing axonal compression. We propose to quantify asymmetry as the Hellinger distance [8] between each EAP and its axially reflected version. We finally present experiments elucidating the link between EAP asymmetry and axonal tortuosity rates, thus compression degrees. Results are shown for EAPs generated analytically in 2D and validated by means of 3D Monte-Carlo simulations performed with Camino [9].

\section{Theory and Methods}

In this section, we introduce a model describing the undulated axon geometry with varying tortuosity. We derive the corresponding equations for the 2D Ensemble Average Propagator (EAP) and discuss its relation with the magnitude and phase of the DW-MRI signal.

\subsection{Tortuous Axon Model}

To analyze the DW signal in axonal compression cases, we model straight axons, in agreement with the usual hypothesis in the area, and undulated axons showing tortuosity. Our model for undulated axons is an extension of that proposed in [4]. We consider a sinusoidal axon that lies in the xz-plane and evolves along $\mathrm{z}$ with oscillations along $\mathrm{x}$. Our axon model has infinitesimal thickness and goes through two full periods within the interval $[-Z, Z]$ with $Z>0$ :

$$
x=f(z)=A \sin \left(\frac{2 \pi(z-Z)}{L(z, \alpha)}\right)
$$

where $A$ and $L(z, \alpha)$ are respectively the amplitude and the wavelength of the sinusoid, with $0<A \leq X$ and $L(z, \alpha)>0$. We model the varying wavelength as

$$
L(z, \alpha)=\alpha(z+Z)+l, \text { for } \alpha>0
$$

where $\alpha$ is the spatial rate regulating the wavelength increment along the $z$ axis. The wavelength increases progressively along the z-axis starting from a minimum value $l$ in $z=-Z$. The spatial rate $\alpha$ is related to the degree of intrinsic asymmetry induced in the undulated shape along the z-axis. We consider the case where $\alpha=0$ as the straight axon, that is when $A=0$, as shown in fig. 1 right. To derive the EAP in the following section it is useful to define the curve length for eq. (1). In particular the length between $z$ and $z+\Delta z$ is given by

$$
s(\Delta z ; z)=\int_{z}^{z+\Delta z} \sqrt{1+\left[A \cos \left(\frac{2 \pi(\zeta-Z)}{L(\zeta, \alpha)}\right) \frac{2 \pi(l+2 \alpha Z)}{L(\zeta, \alpha)^{2}}\right]^{2}} d \zeta
$$

where $\Delta z$ is a displacement along the z-axis with respect to the coordinate $z$. 


\subsection{Ensemble Average Propagator for Tortuous Axons}

To obtain the complex DW signal in the case of tortuous axons model in section 2.1, we derive the correspondent Ensemble Average Propagator (EAP). The EAP for a spin displacement $(\Delta x, \Delta z)$ subject to a diffusion time $t_{d}$ is [6]

$$
\begin{aligned}
\operatorname{EAP}\left(\Delta x, \Delta z ; t_{d}\right) & =\iint_{-\infty}^{\infty} \rho_{0}(x, z) P\left(\Delta x, \Delta z \mid x, z, t_{d}\right) d x d z \\
& =\int_{-\infty}^{\infty} \rho_{0}(z) P\left(\Delta x, \Delta z \mid z, t_{d}\right) d z
\end{aligned}
$$

where $\rho_{0}$ is the initial spin density and $P$ is the displacement pdf. The second equality in eq. (4) is due to our model parametrization in eq. (1). We compute $\rho_{0}$ by assuming an initial uniform spin density along our axon model

$$
\rho_{0}(z)=\cos \left(\arctan \left[f^{\prime}(z)\right]\right)^{-1}
$$

where $f^{\prime}(z)$ is the derivative of $f$ in eq. (1). To produce an approximation for the displacement probability $P$ in eq. (4), we decompose it using:

$$
P\left(\Delta x, \Delta z \mid z, t_{d}\right)=P(\Delta x \mid \Delta z, z) P\left(\Delta z \mid z, t_{d}\right) .
$$

Using this decomposition, we assume that spins diffuse freely along the curve, i.e. they follow a normal distribution with variance $2 D t_{d}$ where $D$ is the selfdiffusion coefficient of water. Then, the probability of a spin experiencing a net displacement along the z-axis $\Delta z$ can be approximated by [4]

$$
P\left(\Delta z \mid z, t_{d}\right)=\frac{1}{\sqrt{4 \pi D t_{d}}} e^{-\frac{s(\Delta z ; z)^{2}}{4 D t_{d}}}
$$

where $s$, the arc length of the axon between $z$ and $z+\Delta_{z}$, is in eq. (3). As the coordinate $\mathrm{x}$ on the axon is univocally determined by $\mathrm{z}$, as specified in eq. (1), the displacement density along $\mathrm{x}$ provided that we know $\Delta z$ and $z$ is [4]

$$
P(\Delta x \mid \Delta z, z)=\delta_{D}(f(z)+\Delta x-f(z+\Delta z))
$$

where $\delta_{D}$ is the Dirac delta function. Given eqs. (5) to (8), the EAP of a tortuous axon, according to our model in section 2.1, can be obtained by simple numerical integration of eq. (4).

We proceed to express the relationship between EAP and signal, then we quintify EAP asymmetry by exploiting the phase information.

\subsection{Diffusion Signal and EAP Asymmetry}

The signal acquired in DW-MRI can be represented as function of the diffusion time $t_{d}$ and frequency vector $\mathbf{q}=\gamma \delta \mathbf{G} / 2 \pi$ where $\gamma$ is the gyromagnetic ratio, $\delta$ is the diffusion pulse duration and $\mathbf{G}$ is the diffusion gradient vector. The 
measured q-dependent signal $S(\mathbf{q})$ has a complex nature and its attenuation $E(\mathbf{q})=S(\mathbf{q}) / S(0)$ is related to the EAP via a Fourier relationship [6]:

$$
E\left(q_{x}, q_{z} ; t_{d}\right)=\iint_{-\infty}^{\infty} \operatorname{EAP}\left(\Delta x, \Delta z \mid t_{d}\right) e^{-j 2 \pi\left(q_{x} \Delta x+q_{z} \Delta z\right)} d \Delta z d \Delta x
$$

which is valid under narrow pulse approximation and where $q_{x}$ and $q_{z}$ are the components of the frequency vector $\mathbf{q}$. The EAP usually calculated from the magnitude attenuation $|E|$ via inverse Fourier transform is symmetric due to the zero phase assumption [10]. We consider instead the complex attenuation and characterize the asymmetry of the diffusion process by measuring the distance between the corresponding EAP and its axially reflected version. Despite different measures are possible, after defining the displacement vector $\mathbf{r}=(\Delta x, \Delta z)$, we adopt the Hellinger distance [8] because it constitutes a proper metric between probability measures

$$
H^{2}=\frac{1}{2} \int\left(\sqrt{\operatorname{EAP}\left(\mathbf{r} \mid t_{d}\right)}-\sqrt{\operatorname{EAP}\left(-\mathbf{r} \mid t_{d}\right)}\right)^{2} d \mathbf{r}
$$

where $0 \leq H \leq 1,0$ corresponding to equality and 1 to maximum inequality.

\subsection{Monte-Carlo Simulation of DW-MRI in Tortuous Axons}

To validate analytic results, we generate the DW complex signal by performing 3D Monte-Carlo simulations using an in-house customized version of Camino [9] that allows to obtain both Magnitude and Phase. This is done by considering both real and imaginary parts of the signal, thus replacing eq. (7) in [9] with

$$
S(\mathbf{G}, \Delta, \delta)=S(0, \Delta, \delta) \int_{-\infty}^{\infty} P(\phi)(\cos (\phi)+j \sin (\phi)) d \phi
$$

where $\phi$ is the phase accumulation, $P(\phi)$ the spin phase distribution and $\Delta$ the time separation between diffusion gradients.

\section{Experiments}

In this section we present experiments showing the relationship between EAP asymmetry and axonal tortuosity for the EAP obtained as described in section 2.2 and with 3D Monte-Carlo simulations.

In the first case we compute the EAP of straight and undulated axons via eq. (4) for increasing tortuosity rates $\alpha \in[1,8]$. Axons present undulation amplitude $A=4 \mu m$ with a basal wavelength $l=50 \mu m$ [4]. We set $D=2 \times 10^{-9} \mathrm{~m}^{2} \mathrm{~s}^{-1}[9]$ and $t_{d}=28.6 \mathrm{~ms}$ according to a realistic scenario. We fix the maximum observable displacement in both directions to $X=Z=50 \mu \mathrm{m}$ and we define the observation frame as the xz-plane limited to the interval $[-X, X]$ along $\mathrm{x}$ and $[-Z, Z]$ along $\mathrm{z}$. As $\alpha$ increases along the z-axis, eq. (2), the undulated axonal trajectory becomes compressed towards the frame's negative bound 


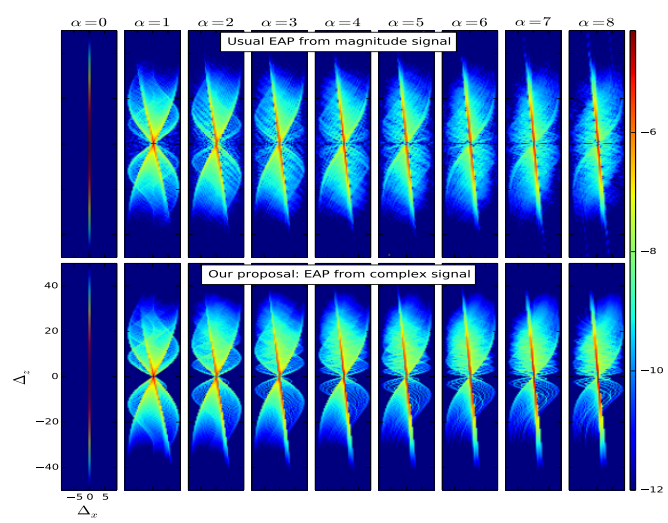

Fig. 2. Log-scaled EAPs obtained for straight and undulated axons with different tortuosity rates $\alpha$. The first row shows EAPs obtained via inverse Fourier transform of the magnitude signal attenuation: EAPs show axial symmetry with respect to the axis passing through the origin. The second row shows EAPs obtained from the complex signal: axial asymmetry is present and increases with the tortuosity rate $\alpha$.

$-Z$ (see fig. 1 right). The observation frame is discretized over a $257 \times 257$ displacement grid, and the EAP is calculated for each sample via numerical evaluation of eq. (4). The complex signal attenuation is obtained, for each considered $\alpha$, as the Fourier transform of the EAP over the displacement grid according to eq. (9). The EAPs are then calculated as the inverse Fourier transform of both the complex and magnitude signal attenuations.

Images in fig. 2 show the log-transformed EAPs calculated for the straight axon $(\alpha=0)$ and for different degrees of axonal compression $(\alpha>0)$. The first row contains the EAPs as usually calculated from the magnitude signal. The second row shows the EAPs obtained from the complex signal. We note that no differences exist for the straight axon $\alpha=0$. Despite the shape of the magnitude-derived EAP changes as $\alpha$ increases, we note that it always shows axial symmetry. On the other end, evident asymmetry is present in the case of the complex-derived EAPs for $\alpha>0$. When looking at the EAPs in the second row of fig. 2, the difference between the upper and lower lobes of each EAP clearly appears. We further note that the degree of asymmetry between the lobes is more marked at high $\alpha$, for instance the difference between the lower and upper lobes for $\alpha=8$ is more accentuated than in the case of $\alpha=1$.

We quantify asymmetry in the EAPs by computing the proposed Hellinger distance, eq. (10), as function of $\alpha$ (fig. 3 left). We note the incremental trend between the complex-derived EAP asymmetry and the tortuosity rate, in agreement with images in the second row of fig. 2 . To validate the results for the more realistic case in which axons have finite diameter, we reproduce experiments using 3D Monte-Carlo simulations. Complex signals are obtained via PGSE sequences with $\Delta=24.3 \mathrm{~ms}, \delta=12.9 \mathrm{~ms}$ and maximum gradient strength $\left|\mathbf{G}_{\max }\right|=0.8 \mathrm{Tm}^{-1}$. Gradient directions are selected over the undersampled 

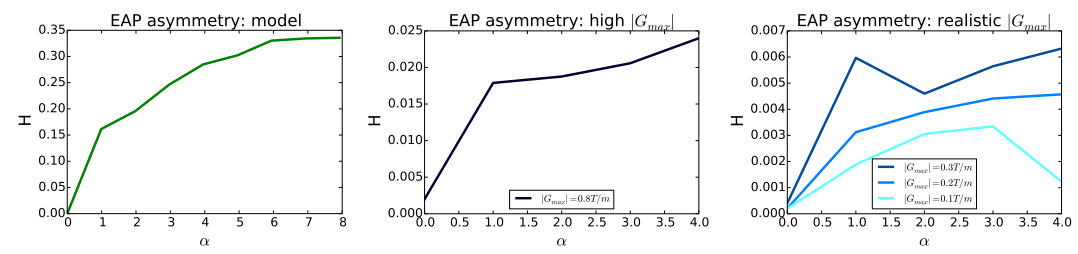

Fig. 3. Hellinger distances as function of tortuosity rate $\alpha$ obtained for the analytic model (left) and via Monte-Carlo simulation (central, right). Right figure shows the behaviour for state-of-the-art human MRI scanners such as the HCP one.

$29 \times 29$ displacement grid. Simulations are carried out considering $10^{5}$ spins diffusing within the axon [9]. We generate meshes for axons with $\alpha=0,1,2,3,4$ considering a diameter $d=1 \mu m$ [4], as shown in fig. 1 right. Meshes have impermeable walls with zero thickness. As for the analytic case we obtain the complex-derived EAPs for increasing $\alpha$ and compute the Hellinger distance to measure the asymmetry, as shown in central fig. 3 . To assess the feasibility of the technique in state-of-the-art human settings, such as in the Human Connectome Project, we explore the behavior of the Hellinger distance at different gradient strengths $\left|\mathbf{G}_{\max }\right|=0.1,0.2,0.3 \mathrm{Tm}^{-1}$, as shown in fig. 3 right.

\section{Discussion and Conclusion}

The overall objective of this work is to assess the use of the DW signal's phase as microstructural marker. We show that the phase becomes informative whenever the underlying diffusion process manifests asymmetry along specific directions as a consequence of, for instance, a variation in the axonal tortuosity.

EAP asymmetry increases with larger tortuosity rates in our $2 \mathrm{D}$ model. This is observable in fig. 3 left and fig. 2 (second row), where values along radial lines passing through the origin are asymmetric for $\alpha>0$. The explanation of this phenomenon lies in the spin displacements asymmetry along the axes. Indeed the maximum distance a spin can travel along an axon evolving along the z-axis is defined by the diffusion coefficient and time. This distance is fixed irrespective of the fact that the spin displaces, along the axon, towards the positive or the negative values of the z-axis. This is the case of the straight axon $(\alpha=0)$ where diffusion occurs equally in each direction (fig. 2). With undulation the maximum displacement along $\mathrm{z}$, resulting from a movement along the axon, depends on the movement direction of the spin, and precisely on the average tortuosity the axon shows in each direction: the ratio between the length of the trajectory and the corresponding length along the z-axis. If the tortuosity in the two directions is different $(\alpha>0)$, then the displacement probability of the spin along the z-axis, thus the $\mathrm{x}$-axis, is asymmetric with respect to the origin. Since the EAP accounts for all of the contributions of the spin displacement probabilities along the axon, the EAP is symmetric when axonal tortuosity is constant $(\alpha=0)$, and is asymmetric otherwise $(\alpha>0)$. 
EAP asymmetry is sensible to axonal tortuosity in realistic scenarios. In 3D experiments spins diffuse transversely to the axon as well as along it. Hence, the trajectory a spin performs is convoluted and leads to smaller displacements along the axon, causing the asymmetry to have a milder effect on the overall EAP. This directly implies that a good characterization of the EAP asymmetry may be better achieved at very high $|\mathbf{q}|$ values as shown in fig. 3 central and right. Lower gradient strengths, hence displacement resolutions, affect the measured asymmetry at very short wavelengths as for the case of $\alpha=4$ and $\left|\mathbf{G}_{\max }\right|=0.1 \mathrm{Tm}^{-1}$. However, our proposed measure of asymmetry using the complex signal combined with the Hellinger distance, eq. (10), as microstructure marker for axonal tortuosity/compression, remains effective. As a future work we suggest to explore higher diffusion times, and thus $\Delta$. While a very high $|\mathbf{q}|$ is hard to achieve, longer diffusion times allow spins to diffuse more thus sensing the asymmetry at greater displacements, which are observable also at lower $|\mathbf{q}|$.

Real data acquisition for this scenario is challenging, specially due to technical difficulties such as the extreme sensibility of phase to noise. However, although other techniques assume straight axons, simulations show that undulation combined with tortuosity significantly affect the DW signal and can be detected through diffusion asymmetry by exploiting the phase. We strongly believe that this pioneering work opens new roads and perspectives towards measuring WM tissue properties via DW-MRI.

\section{References}

1. Assaf, Y., Blumenfeld-Katzir, T., Yovel, Y., Basser, P.J.: AxCaliber: a method for measuring axon diameter distribution from diffusion MRI. MRM, 59(6), 1347-1354 (2008)

2. Zhang, H., Schneider, T., Wheeler-Kingshott, C.A., Alexander, D.C.: NODDI: practical in vivo neurite orientation dispersion and density imaging of the human brain. Neuroimage, 61(4), 1000-1016 (2012)

3. Alexander, D.C.: A general framework for experiment design in diffusion MRI and its application in measuring direct tissue-microstructure features. MRN, 60(2), 439448 (2008)

4. Nilsson, M., Lätt, J., Stahlberg, F., Westen, D., Hagsltt, H.: The importance of axonal undulation in diffusion MR measurements: a Monte Carlo simulation study. NMR in Biomedicine, 25(5), 795 (2012)

5. Shacklock, M., Biomechanics of the Nervous System: Breig Revisited. Neurodynamics Solutions, Adelaide, (2007)

6. Tanner, J.E., Stejskal, E.: Restricted Self-Diffusion of Protons in Colloidal Systems by the Pulsed-Gradient, Spin-Echo Method. JCP, 49(4), 1768-1777 (1968)

7. Özarslan, E., Koay, C.G., Basser, P.J.: Remarks on q-space MR propagator in partially restricted, axially-symmetric, and isotropic environments. MRI, 27(6), 834-844 (2009)

8. Hellinger, E.: Neue Begründung der Theorie quadratischer Formen von unendlichvielen Veränderlichen. Journal für die reine und angewandte Mathematik (1909)

9. Hall, M.G., Alexander D.C.: Convergence and parameter choice for Monte-Carlo simulations of diffusion MRI. IEEE TMI, Vol. 28, pp. 1354-1364 (2009)

10. Bracewell, R.: The Fourier transform and its applications. New York (2000) 